\title{
Pour une sociologie de l'objet mondialisation
}

Jean-Luc Metzger

\section{OpenEdition}

\section{Journals}

Édition électronique

URL : http://journals.openedition.org/ress/516

DOI : $10.4000 /$ ress.516

ISSN : 1663-4446

\section{Éditeur}

Librairie Droz

\section{Édition imprimée}

Date de publication : 1 décembre 2003

Pagination : 173-192

ISBN : 2-600-00912-4

ISSN : 0048-8046

Référence électronique

Jean-Luc Metzger, «Pour une sociologie de l'objet mondialisation », Revue européenne des sciences sociales [En ligne], XLI-127 | 2003, mis en ligne le 30 novembre 2009, consulté le 07 mai 2019. URL: http://journals.openedition.org/ress/516; DOI : 10.4000/ress.516 
Jean-Luc METZGER

\title{
POUR UNE SOCIOLOGIE DE L'OBJET MONDIALISATION
}

\begin{abstract}
Je me suis efforcé de saisir la pensée de ces sociologues, sans méconnaître ce que nous considérons comme l'intention spécifique de la sociologie, sans oublier non plus que cette intention était inséparable, au siècle dernier, des conceptions philosophiques et d'un idéal politique. Peut-être d'ailleurs n'en va-t-il pas autrement chez les sociologues de notre temps, dès qu'ils esquissent une interprétation globale de la société ${ }^{\text {. }}$
\end{abstract}

\section{INTRODUCTION}

On ne compte plus les publications, «savantes» ou «profanes», dont le titre comprend le terme mondialisation. L'examen des catalogues des éditeurs montre que, depuis 1995, les productions sur ce thème sont chaque année plus abondantes. Pourtant, certains auteurs ${ }^{2}$ soulignent le paradoxe d'une situation où $l$ ' explosion de la communication ${ }^{3}$, loin d'être un atout pour comprendre le monde, brouille au contraire son appréhension, tant les «innovations » sont dorénavant livrées avec le discours censés en livrer les clés. Et s'il est urgent de clarifier la notion de mondialisation ${ }^{4}$, ainsi que la réalité qu'elle entend désigner, il nous semble également capital de montrer que cet effort de prise de distance relève du champ de compétences de la sociologie, voire de la tradition sociologique.

En effet, si l'on admet que notre monde se caractérise par la tension entre des forces d'homogénéisation et de différenciation, alors «l'examen d'une telle question s'inscrit dans une longue tradition de recherche», propre aux sciences sociales 5 . Mais, parmi toutes les sciences sociales, quelle est la «légitimité de la sociologie lorsqu'elle s'aventure sur le vaste terrain de la mondialisation»? «Est-elle équipée pour analyser, interpréter et comprendre cet objet d'étude, alors qu'elle a plutôt l'habitude de ne s'intéresser qu'à des terrains plus limités » ${ }^{6}$ ?

\footnotetext{
R. Aron, 1967, p. 17.

C'est notamment le cas de A. Mattelart (1996) et de F. Cooper (2001).

3 Selon le titre du livre de P. Breton et S. Proulx, 1989, L'explosion de la communication. La naissance d' une nouvelle idéologie, La Découverte, 1995.

4 Comme s'y emploie S. Latouche (2001), pour qui le terme globalization aurait été lancé au début des années 80 par Sony, pour la vente des baladeurs et aurait été repris par les firmes transnationales et le gouvernement américain. G. Busino (2001) soutient, quant à lui, que les termes de mondialisation et de globalisation datent de la fin des années 1950. Voir également D. Martin, J.-L. Metzger et P. Pierre (2003), notamment le premier chapitre.

5 D. Mercure, 2001, p. 9.

6 G. Rocher, 2001.
} 
Pour répondre, commençons par poser qu'analyser sociologiquement la mondialisation équivaut à appliquer les catégories de cette discipline dans trois perspectives distinctes et complémentaires:

$1^{\circ}$ ou bien, l'observateur veut rendre compte d'une réalité sociale d'extension planétaire (l'objet-monde). L'ensemble des sociétés est alors envisagé comme un tout et les entités supranationales (institutions, entreprises, associations, élites) sont au cœur de l'analyse;

$2^{\circ}$ ou bien, il s'intéresse aux transformations qui touchent les différentes dimensions sociales (culture, économie, politique, etc.) des différentes sociétés, en comparant ces transformations pour identifier leur degré de convergence et d'interdépendance. Ce qui renvoie à l'analyse du changement social dans une perspective planétaire (objet-mondialisation);

$3^{\circ}$ ou bien, enfin, il centre son intérêt sur l'articulation entre les transformations macrosociales et celles affectant le champ de la sociologie, de manière à identifier les influences mutuelles et ainsi s'affranchir de l'illusion de l'extériorité savante.

Comment ces trois perspectives ont-elles été envisagées jusqu'à présent en sociologie? En répondant à cette question, sans prétendre pour autant à l'exhaustivité nous chercherons à dégager des outils utiles pour comprendre la mondialisation.

\section{ANALYSER L'OBJET-MONDE : RENOUER AVEC LA TRADITION SOCIOLOGIQUE}

Comme le note A. de Swaan (1998), les précurseurs de la sociologie (Smith et Marx) n'assimilaient pas la notion de société à celle d'Etat-Nation, mais à celle d'humanité (la richesse, pour A. Smith était celle des nations, le capitalisme pour Marx était d'emblée planétaire). Dès lors, analyser un objet dépassant le cadre des frontières (la société transnationale) c'est bien renouer avec la vocation originelle de la sociologie.

\section{La société-monde comme horizon de la démarche sociologique}

Dans le même sens, E. Durkheim (1978a) rappelle que l'œuvre d'un autre précurseur de la sociologie, Saint-Simon, était toute tendue vers l'appréhension de l'international. En effet, pour Saint-Simon, la société industrielle doit engendrer un type d'organisation qui ne peut se limiter à une société particulière et «tend à prendre une forme internationale». Saint-Simon envisage les faits sociaux, d'emblée à l'échelle européenne, et évoque l'extension de la société industrielle à l'humanité. Ainsi, l'un des journaux fondés par les saint-simoniens s'appellera Le Globe.

Rappelons encore que les fondateurs de l'Ecole française de sociologie (E. Durkheim et ses successeurs), mais également les sociologues allemands de la fin

En effet, notre objectif n'est pas, dans le cadre de cet article, de procéder à un recensement complet des travaux «classiques» portant sur la société-monde, mais simplement de souligner l'antériorité de sa conceptualisation. 
du $\mathrm{XIX}^{\mathrm{e}}$ siècle, ont souvent procédé, pour rendre compte des phénomènes sociaux, à des analyses comparatives, tant entre différentes sociétés contemporaines, qu'entre sociétés appartenant à des époques différentes. Par ces comparaisons, ils cherchaient à identifier les caractéristiques communes à plusieurs groupements humains, considérées comme universelles. Et ce qui est au minimum conçu comme proprement universel, c'est la propension des sociétés, au fur et à mesure de leur développement, à connaître une division interne des activités et de l'organisation, ainsi que la nécessité de compenser le manque de solidarité qui en résulte.

La plupart de leurs travaux appellent deux types de remarques. D'une part, pour pouvoir effectuer ces comparaisons, les chercheurs ont admis (explicitement ou implicitement) l'homologie inter-temporelle et inter-nationale des formes sociales. Ce qui constitue une première dimension de l'appréhension du social comme entité d'emblée globale. Au fond, celui-ci, au-delà de son infinie diversité, était a priori décomposable en éléments simples (les structures élémentaires, les formes élémentaires), classables en des catégories universelles (la famille, la hiérarchie, la religion, la solidarité, le conflit, le pouvoir légitime, etc.).

D'autre part, leur interprétation est sous-tendue par une conception globalement progressiste de l'évolution sociale, celle-ci se faisant, en quelque sorte à l'échelle de la planète: telle société reprenant, à une époque donnée, le flambeau de l'évolution, avant de la passer à la suivante, le tout débouchant sur un perfectionnement de l'humanité. «En un mot, on ne peut admettre que le progrès ne soit qu'un effet de l'ennui. Cette refonte périodique et même, à certains égards, continue de la nature humaine, a été l'oeuvre laborieuse qui s'est poursuivie dans la souffrance. Il est impossible que l'humanité se soit imposé tant de peine uniquement pour pouvoir varier un peu ses plaisirs et leur garder leur frâ̂cheur première. ${ }^{8}$ Dit autrement, pour les fondateurs, le regard sociologique doit reconstituer un métadéveloppement, au-delà des frontières du temps et de l'espace.

Les références, sans être abondantes, n'en sont pas moins explicites qui montrent que Durkheim avait identifié la question de la société-monde: «c'est un rêve depuis longtemps caressé par les hommes que d'arriver enfin à réaliser dans les faits l'idéal de la fraternité humaine. Les peuples appellent de leur vœux un être où (...) tous les hommes collaboreraient à la même œuvre et vivraient de la même vie. (...) [Ces aspirations] ne peuvent être satisfaites que si tous les hommes forment une même société, soumise aux mêmes lois. Car, (...) les conflits inter-sociaux ne peuvent être contenus que par l'action régulatrice d'une société qui comprenne en son sein les autres ${ }^{9}$. Même si, pour le moment (au début du $\mathrm{XX}^{\mathrm{e}}$ siècle), «il faut bien reconnaître que cet idéal n'est pas à la veille de se réaliser intégralement (...). Ce qui est possible, c'est que les sociétés de même espèce s'agrègent ensemble, c'est bien dans ce sens que parât se diriger notre évolution ».

Mais cette perspective, qui semble de nos jours prendre corps dans le projet de communauté européenne, n'est pas sans effrayer: "Alors, qu'est-ce qui se produira à la limite? Si toutes les patries se confondaient en une seule, celle-ci resterait une patrie, car ce serait une société organisée. Pourtant, une patrie unique

E. Durkheim, 1978b, p. 236.

9 E. Durkheim, 1978b, p. 401. 
est-elle concevable? Le moi ne se pose qu'en s'opposant. (...) Il n'y aurait plus qu'une conscience pour penser le monde.»10

L'auteur semble rassuré qu'il n'existe pas de groupement politique plus étendu que l'Etat et que «l'humanité, dans son ensemble, n'est qu'un être de raison. Ce qui existe réellement ce sont des sociétés particulières (les tribus, les nations, les cités, les Etats de toutes sortes, etc.), qui naissent et qui meurent, qui progressent et régressent (...) sans que ces évolutions diverses soient toujours sur le prolongement les unes des autres ${ }^{11}$. La notion d'humanité semble plutôt constituer le terme ultime d'une série (au sens mathématique) qui renforce la thèse sur le social.

Successeur de Durkheim, M. Mauss a apporté sa contribution à l'identification de l'objet-monde, en réfléchissant à la question de la nation, des nationalités, de l'internationalisme. «Mais quel genre de société mérite le nom de nation?» ${ }^{12}$. La réponse que donne l'auteur s'inscrit dans la conception évolutionniste des sociétés: seules celles qui ont atteint un stade de développement suffisant (notamment un degré d'intégration) méritent d'être classées dans cette catégorie (implicitement considérée comme désirable). Toutes les autres en sont restées à des étapes intermédiaires, pour ainsi dire préliminaires. Au-delà de cette posture, l'auteur souligne que le mouvement d'expansion des nations «a abouti non pas à une uniformisation de la civilisation, mais, à certains points de vue, à une individualisation de plus en plus profonde des nations et des nationalités ${ }^{13}$. En d'autres termes, Mauss avait déjà conscience du caractère paradoxal de l'évolution sociale: la diffusion d'un même modèle de société s'accompagne d'un accroissement des particularismes.

Poursuivant la réflexion sur cet axe, l'auteur en vient à analyser les phénomènes internationaux, les relations entre nations qui «ne sont qu'un cas des relations entre sociétés ». L'élaboration d'une société-monde n'est pas impensable: «la formation de groupes de plus en plus vastes, absorbant des nombres de plus en plus considérables de grandes et de petites sociétés est une des lois les mieux constatées de l'histoire. De telle sorte qu'il n'y a aucune limite à faire a priori à ce processus $\gg^{14}$. Le travail du sociologue est alors de «montrer à quel point de cette évolution l'humanité est parvenue aujourd'hui ». D'ailleurs, au début des années 1920, Mauss pense «que l'on peut parler maintenant de civilisation humaine mondiale, pour employer un mot du jargon moderne $»^{15}$. L'auteur parle explicitement d'interdépendance de chaque économie nationale avec «une certaine tonalité du marché mondial, en particulier de celui des métaux précieux, étalons de valeurs». Ce qui préfigure clairement l'intérêt, que l'on croit trop souvent récent, pour les marchés financiers.

Cet attrait pour les questions internationales, non seulement rappelle l'antériorité de la problématique en sociologie, mais surtout permet de développer une certaine conception du social où celui-ci, même lorsqu'on ne le considère que d'un

10 E. Durkheim, 1975, t. 3, pp. 222-223. On ne peut s'empêcher de confronter cette réflexion à la situation contemporaine où l'extension de la puissance d'une nation semble donner à la pensée de Durkheim la forme d'une intuition prémonitoire.

11 E. Durkheim, 1975, t. 1, p. 111.

12 M. Mauss, 1969, p. 578.

13 M. Mauss, 1969 , p. 591

14 M. Mauss, 1969, p. 606.

15 M. Mauss, 1969, p. 610. 
point de vue strictement local, résulte d' «un état de perméation et de dépendance mutuelle croissante». En d'autres termes, pour comprendre ce que l'on observe localement, empiriquement, il faut prendre en compte les contextes macro-sociaux, les relations avec le global. «Ce sont précisément ces relations entre sociétés qui sont explicatives de bien des phénomènes de la vie intérieure des sociétés ». Ainsi, «maintenant, l'ockoumène forme un monde, (...) la suite des événements va dans le sens d'une multiplication croissante des emprunts, des échanges, des identifications, jusque dans le détail de la vie morale et matérielle ${ }^{16}$.

Loin de n'aboutir qu'à un intérêt anecdotique pour l'objet virtuel que serait l'humanité, cet effort permet à l'auteur d'identifier deux «cercles » d'interdépendances contraignantes, l'un en matière de maîtrise des techniques, l'autre concernant les institutions internationales. En effet, les grandes nations n'ont pratiquement plus le choix de refuser de s'emprunter mutuellement des innovations scientifiques et techniques, puisqu'elles constituent la source indispensable de l'indépendance militaire. On serait tenté de retrouver, dans cette conception, le point de vue contemporain consistant à affirmer le caractère inéluctable du mouvement vers plus de compétition, de flexibilité, mais aussi d'accords juridiques internationaux. En effet, grâce aux conventions internationales, les nations reconnaissent l'existence de ces phénomènes et manifestent la volonté de les légitimer (et, sans doute, de les contrôler). Mais simultanément, une fois conclues, ces conventions conduisent «les différents Etats à adopter au fond les mêmes principes de droit et mieux des jurisprudences identiques $\gg^{17}$.

En somme, si l'objet-monde n'était pas, à proprement parler, au centre des préoccupations des fondateurs de la sociologie, ils ont néanmoins adopté une posture et forgé des concepts suffisamment généraux pour aborder la sociétémonde de deux manières:

- au sens abstrait du terme, comme l'arrière-plan qui sous-tend la sociologie, comme le terme d'un raisonnement qu'on extrapole pour en montrer la puissance;

- empiriquement, comme principe général de comparaison des sociétés concrètes.

\section{L'économie-monde comme horizon de la rationalisation}

C'est sans doute dans son Histoire économique ${ }^{18}$ que Max Weber envisage avec le plus de clarté le social comme un tout dont on peut reconstituer la genèse en comparant les sociétés à travers les siècles et les continents ${ }^{19}$. Dans cette somme d'érudition, l'auteur mobilise en effet un grand nombre de données (politiques, économiques, sociales, culturelles, religieuses) correspondant à plusieurs

16 M. Mauss, 1969, p. 625.

17 M. Mauss, 1969, p. 619.

18 M. Weber, 1991 [1923]. Le sous-titre complet est: Histoire économique. Esquisse d'une théorie universelle de l'économie et de la société. L'auteur a donc bien comme ambition d'atteindre à l'universel, envisagé à la fois comme englobant les millénaires et les continents, mais également comme évolution éventuellement commune à l'humanité.

19 Comme le note P. Raynaud (1987, p. 65), cette volonté est générale à toute 1 'œuvre de Max Weber. 
aires de civilisation (Europe, Amérique, Chine, Inde, monde Arabe), afin d'identifier les raisons pour lesquelles le capitalisme n'a émergé que dans un petit nombre de nations. Il définit l'évolution (trans-historique et méta-sociétale) comme, avant tout, un processus de rationalisation, concernant certes le calcul économique, mais également «des éléments de type extra-économiques. Notamment, les aspects magiques et religieux (...), les aspects politiques - la soif du pouvoir -, les intérêts liés à la position sociale - la recherche des honneurs $»^{20}$.

Max Weber, en empruntant des «moments d'histoire» et des «fragments de sociétés », se réfère implicitement à une méta-société - l'humanité -, dont, à chaque période, les différents groupements régionaux constitueraient les composantes. Qu'il s'agisse du droit, de la technologie, des finances («la monnaie et l'histoire de la monnaie $»^{21}$ ), du travail ( «la situation des paysans dans chacun des pays occidentaux avant l'émergence du capitalisme»), ou encore des relations internationales ( «la politique coloniale du XVI ${ }^{\mathrm{e}}$ au XVIII ${ }^{\mathrm{e}} »$ ) ou des pratiques religieuses, la succession de crises et d'inventions, caractéristiques des différentes sociétés locales, par emprunts ou par la guerre, par imitation ou par anticipation, permet une sorte d'apprentissage à l'échelle des continents ou des ensembles de continents. Plus précisément, l'auteur tend à identifier, non comment des traits constitutifs de la société capitaliste moderne se seraient développés et auraient diffusé, mais plutôt quels obstacles en ont empêché l'émergence ou l'adoption en Chine, en Inde ou dans l'Antiquité. De ce point de vue, l'obstacle le plus conséquent réside dans le maintien des croyances magiques ${ }^{22}$.

Max Weber contribue, d'une autre manière, à l'analyse de la société-monde, ou plus exactement de certaines dimensions de la mondialisation économique. L'auteur, en effet, aborde la question de l'internationalisation des firmes (1991, chapitre 4). Il souligne deux éléments structurant ce développement: le rôle de l'Etat pour en faciliter la genèse; et l'extension préalable du «mode de pensée capitaliste», lequel permet d'envisager comme rationnel de couvrir tous les besoins quotidiens de manière capitaliste.

De même, W. Sombart, dans L'apogée du capitalisme ${ }^{23}$, analyse le développement des entreprises modernes en termes de rationalisation. Et ce, selon deux axes : la concentration financière des «firmes en réseau » qui permet d'obtenir des interdépendances, lesquelles ont «pour effet d'imposer à toutes les entreprises associées une seule volonté, une direction commune et un plan d'ensemble »24; et l'organisation scientifique du travail qui introduit « un système d'opérations artificielles rattachées les unes aux autres et exécutées par des fonctionnaires interchangeables, n'ayant d'humain que la forme et l'aspect extérieurs » ${ }^{25}$. Grâce à ces

\footnotetext{
20 M. Weber, 1991, p. 26

21 Dans cette catégorie, on peut inclure La philosophie de l'argent de G. Simmel (1987 [1900]), où l'auteur reconstitue, à partir de nombreux exemples empiriques empruntés à différentes civilisations d'époques différentes, le mouvement général, universel, par lequel l'argent qui, sous ses différentes formes, s'est peu à peu dégagé de sa matérialité pour symboliser la pure valeur économique.

22 M. Weber, 1991, p. 386.

23 G. Sombart, 1932. Essentiellement la troisième subdivision: «la rationalisation des entreprises » pp. 215-456.

24 G. Sombart 1932 p. 251.

25 G. Sombart, 1932, p. 251 et p. 404.
} 
deux rationalisations combinées, la localisation géographique des procédés productifs est sans importance. Le «système» et «l'organisation » fournissant des prescriptions abstraites, préexistant à toute opération, fonctionnant dans un mouvement automatique, remplacent l'individu et sa personnalité. Comment alors s'étonner que de nationale, l'entreprise devienne internationale? Cette extension nécessité toutefois, comme dans l'approche de Weber, l'intervention d'un Etat entrepreneur. Pour Sombart (1966), l'Etat fournit une sorte de prototype des grandes entreprises capitalistes: la rigueur de son organisation, la hiérarchie de ses fonctionnaires, sa persévérance à atteindre ses fins, offrent autant d'exemples à suivre chez les chefs d'entreprise. De plus, en favorisant un certain type d'enseignement, il contribue à la diffusion préalable d'un état d'esprit, d'un mode de pensée que Sombart qualifie de bourgeois.

Soulignant à la fois le rôle d'acteurs privilégiés (entrepreneurs, décideurs publics) et le caractère quasi-automatique de l'extension internationale des groupements sociaux, insistant sur l'interdépendance des dimensions économiques, culturelles et imaginaires, les premiers sociologues allemands ont développé un système de représentations qui sous-tend encore notre façon de penser les transformations actuelles. Parmi ces représentations, notons, avec J.C. RuanoBorbalan (2001), la sous-estimation de la dimension militaire, violente, prédatrice, pour expliquer l'extension de la rationalité occidentale, celle-ci semblant émerger de la nature des relations sociales.

\section{La société internationale comme espace de luttes pour l'hégémonie}

Dans les années 1930-1950, Norbert Elias s'est attaché à comprendre également le processus de transformation à l'œuvre dans les sociétés occidentales, depuis le Moyen-Age ${ }^{26}$, en centrant son analyse sur le développement conjoint de l'Etat absolutiste (mû par la compétition entre unités de dominations) et de l'individu moderne (par élaboration de schèmes d'autocontrainte).

Schématiquement, pour Elias, tandis que des Etats de plus en plus centralisés et vastes se bâtissaient, un modèle de comportement s'est développé et affiné au sein des couches dirigeantes (système d'autocontraintes et de codes visant, d'abord, à assujettir les pulsions), pour aboutir, au XVII' siècle, à son apogée puis s'est diffusé dans toute la société nationale, de couche sociale en couche sociale, moyennant le recours à des forces de l'ordre publiques (monopole de l'exercice de la violence entre les mains de l'Etat). La Révolution française amplifia ce phénomène de diffusion, dans la mesure où elle visait à harmoniser les comportements d'individus dispersés sur de vastes territoires. Puis «les modèles de comportement de la «bonne compagnie» aristocratique ont gagné la vaste société industrielle, même là où les cours étaient moins riches et moins puissantes $»^{27}$. L'extension du modèle de société ouest-occidental se serait ainsi effectué par les groupes dirigeants nationaux, pour aboutir à une «dépendance entre un nombre croissant d'individus, d'espaces, de pays ».

26 Nous faisons référence à N. Elias, 1973, 1975 et 1993. L'auteur s'est surtout appuyé sur une étude sociohistorique de l'Allemagne, de la France et de la Grande-Bretagne.

27 N. Elias, 1975, p. 283. 
Les modèles de comportement occidentaux ont ensuite été étendus à d'autres continents et, plus généralement, aux relations inter-étatiques. Les puissances occidentales ont en effet cherché à "'civiliser' les peuples colonisés (...) [et à] recourir au façonnage du Surmoi pour maintenir un empire ${ }^{28}$. Sur le plan des institutions, la dynamique de compétition entre unités de domination conduit à la lente victoire d'une seule unité: «s'il est vrai qu'il s'agit pour le moment d'abord d'une domination limitée à quelques continents, on voit se dessiner déjà, par suite du débordement des interdépendances sur d'autres régions, la lutte pour l'hégémonie dans un système englobant toute la terre habitée. $\gg^{29}$

Elargissant à l'époque actuelle les conclusions de son étude, N. Elias entend avoir montré combien était illusoire la prétention à ériger la concurrence comme un principe définitif: elle conduit nécessairement au monopole. Qu'il s'agisse du domaine économique, politique, ou de la conquête colonisatrice, le monde s'acheminerait vers un contrôle unique. Car, de même que l'entrepreneur capitaliste en situation de concurrence n'a pas le choix d'agrandir ou non son affaire ${ }^{30}$, de même, «les Etats rivaux se poussent réciproquement dans les remous de la compétition sous la pression de l'ensemble du réseau d'Etats dont ils constituent les éléments ${ }^{31}$. Considérant que «les tensions dues à la concurrence entre Etats ne peuvent s'apaiser», l'auteur anticipe sur la formation de «monopole militaire et policier dépassant le cadre des Etats », devant aboutir à «la mise en place d'un monopole mondial de la force publique, d'un organe politique central $\aleph^{32}$.

Toujours dans les années 1930-1950, le fonctionnalisme et le structuro-fonctionnalisme de T. Parsons et R. K. Merton, fournissent des outils d'analyse abstraits des «sociétés globales ${ }^{33}$, compatibles avec le projet de rendre compte d'une société-monde, connaissant des variantes locales ou historiques. Mais l'ont-ils pour autant envisagé en ces termes? On peut en trouver des traces dans certaines allusions: «la société [nationale] n'est pas le système social le plus vaste mais n'est qu'un élément d'un système supranational plus vaste ${ }^{34}$. Ou encore quand Parsons s'interroge sur le devenir des sociétés nationales et qu'il pense déceler une extension des traits de la modernisation occidentale: «la tendance à la modernisation est devenue mondiale de nos jours (...) [et] se poursuivra probablement dans le monde non-occidental ${ }^{35}$. On retrouve là un intérêt pour l'international proche de celui de Weber, pour qui, selon Parsons, «ce qu'il a appelé la société occidentale de l'ère moderne a une signification 'universelle' dans l'histoire humaine ${ }^{36}$. La réalité

\footnotetext{
28 N. Elias, 1975, p. 290.

$29 \quad$ N. Elias, 1975, pp. 300-301.

30 Accessoirement, N. Elias évoque la constitution «d'organisations monopolistiques privées (...) qui semblent tendre vers une fusion sur le plan de l'organisation» (1975, p. 305), laissant ainsi entrevoir l'existence de monopoles mondiaux, par domaine d'activité, monopoles que l'auteur déconseille de laisser aux seules mains d'initiatives privées.

$31 \quad$ N. Elias, 1975, p. 303.

32 N. Elias, 1975 , p. 317

33 Toute société globale, quelle que soit sa taille, son degré d'organisation, se caractérise par le fait d'avoir mis au point des solutions adaptées pour remplir les quatre fonctions sociales (définies de façon très abstraite par T. Parsons) jugées indispensables et universelles.

34 T. Parsons, 1973a, p. 2.

35 T. Parsons, 1973b, p. 147.
} 
sociale ne prend son sens, ne livre sa signification qu'à l'échelle macro-sociale et historique. Parsons, revendiquant une position néo-évolutionniste, soutient que la «variation» la plus probable que vont connaître toutes les sociétés nationales, consiste en un rapprochement avec le modèle nord-américain.

Contre cette croyance, Raymond Aron cherche à analyser, au-delà du clivage existant alors entre capitalisme et socialisme ( «deux espèces d'un même genre»), «la société internationale telle que l'a faite la modernité ${ }^{37}$, et veut retrouver les caractères communs à toutes les économies de notre époque. Cette posture ne lui fait pas pour autant ignorer combien, « de toute évidence, il n'y a pas d'ordre social de la modernité», dès lors que l'on accepte de distinguer, «à l'intérieur d'une société complexe, de multiples 'cultures', d'un village ou d'un pays à un autre, d'une classe d'âge ou d'une classe sociale à une autre ${ }^{38}$. Mais le développement de cette civilisation rend plus étroites les relations entre les sociétés et plus insupportables les inégalités de richesses entre pays. Au point qu'il est légitime de se demander si la classe ouvrière tend à une homogénéité croissante ou à une hétérogénéité accrue ${ }^{39}$. Car, pour comprendre le social, il faut se situer à l'échelle de la société-monde: «grâce aux possibilités techniques, la planète entière (...) constitue le champ intelligible de l'Histoire.» C'est de ce point de vue que sautent aux yeux les écarts de développement, la pluralité des conditions, le caractère «scandaleux » des choix collectifs et publics d'affectation des ressources. C'est, plus généralement, comme le souligne G. Busino (1986), de ce point de vue que la compréhension des différentes totalités sociales prend son véritable sens, que l'autonomie relative du social, de l'économique et du politique apparaissent clairement.

C'est sans doute dans Paix et guerre entre les nations que R. Aron aborde le plus directement l'analyse sociologique de la société-monde. Ainsi, la présentation à la huitième édition s'intitule «La société internationale»: «peut-être peut-on appeler société internationale ou société mondiale l'ensemble qui englobe le système inter-étatique, l'économie mondiale (ou le marché mondial ou le système économique mondial), les phénomènes transnationaux et supranationaux. (...) Je ne pense pas que la formule société internationale ou, de préférence, mondiale, constitue un véritable concept. (...) Peut-on appeler société cette sorte de totalité qui ne garde presque aucun des traits caractéristiques d'une société, quelle qu'elle soit? (...) J'en doute $»^{40}$.

Certes, R. Aron va privilégier l'analyse des relations entre Etats, considérés comme des acteurs collectifs, au sein desquels la prise de décision demeure complexe. Mais il reconnaît que «le système inter-étatique ne se confond pas avec la société internationale. Il représente un aspect particulier de cette société». D'autres dimensions sont également constitutives de la société internationale: le marché mondial capitaliste (au sein duquel d'ailleurs, les Etats communistes sont partie prenante), ainsi que les croyances et les idéologies. Toutefois, c'est le système inter-étatique «qui structure la société internationale en dépit des traits

\footnotetext{
T. Parsons, 1973b, p. 149.

R. Aron, 1969, p. 310.

R. Aron, 1969, p. 328.

R. Aron, 1964, p. 12.

40 R. Aron, 1984, p. VIII.
} 
originaux de celle-ci: la peur qu'inspirent aux dirigeants politiques l'éventualité de la grande guerre, l'amplification des moyens marginaux de la lutte entre les Etats et, simultanément, la multiplication des échanges entre les sociétés et l'apparition d'une économie transnationale $»^{41}$.

Pour R. Aron, qui rejoint ici Norbert Elias, «tant que l'humanité n'aura pas accompli son unification dans un Etat universel, il subsistera une différence essentielle entre politique intérieure et politique étrangère. Celle-là tend à réserver le monopole de la violence légitime aux détenteurs de l'autorité, celle-ci accepte la pluralité des centres des forces armées $»^{42}$. Certes, on peut envisager une sorte d' «utopie de la confédération ou fédération planétaire ». Mais cela nécessiterait que les individus aient développé «des relations comparables à celles qui lient entre eux les membres (...) de chaque société nationale: conscience de communauté, consentement à un régime juridique et politique, monopole de la force armée $»^{43}$. Ce dont R. Aron doute.

En somme, si, pour R. Aron, la société-monde ne constitue pas un objet d'étude empirique, y faire référence comme à une sorte d'objet ultime, d'horizon de l'analyse, présente plusieurs attraits. Elle permet, tout d'abord, d'accepter d'envisager le social comme traversé de contradictions, en un mot dialectique: «la société de type industriel ne comporte d'intelligibilité que dans et par son mouvement $\gg^{44}$. Mais il s'agit d'un mouvement à l'issue imprévisible, non d'une évolution toute tracée. La référence à l'objet-monde permet ensuite de prendre conscience de multiples effets souvent contradictoires, résultants de ce mouvement: si des communautés de savants sont transnationales, les progrès techniques demeurent possédés et mis en œuvre par des nations rivales; la science paraît reposer sur des valeurs universelles, mais les volontés locales de non-dépendance demeurent. Faire référence à l'humanité permet encore de souligner que, si toutes les sociétés d'un certain type ont des croyances communes (par exemple que l'essentiel réside dans la recherche de puissance et de richesse), cela ne recouvre pas l'univers des possibles: d'autres choix pourraient être faits qui permettraient de «concevoir une société humaine moins soucieuse d'accroître le potentiel de ses machines ou de ses forces productives que d'assurer à tous le minimum nécessaire pour une existence décente $»^{45}$.

Contemporain de R. Aron, Georges Gurvitch (1968) fournit un autre exemple d'intérêt pour la société-monde, et ce, de deux manières. D'une part, par la notion de type de société globale («macrocosmes de groupements particuliers et de classes sociales »), notion par laquelle l'auteur cherche à classer les différents groupements sociaux, tels qu'ils existent ou ont existé de par le monde. On retrouve la volonté de fournir une explication globale du social (méta-social et inter-temporel). Et d'autre part, d'un point de vue empirique, G. Gurvitch consi-

\footnotetext{
R. Aron, 1984, p. XXXVII.

R. Aron, 1984, p. 19.

R. Aron, 1984, p. 738.

R. Aron, 1969, p. 15.

45 R. Aron, 1969, p. 286. L'auteur rencontre ici la sociologie du développement (voir Y. Goussault et A. Guichaoua, 1993) dont il faut rappeler que les préoccupations rejoignent la sociologie du monde et de la mondialisation.
} 
dère que ce qui caractérise la seconde moitié du $\mathrm{XX}^{\mathrm{e}}$ siècle, c'est «la lutte engagée, mais non résolue, entre les types différents de structures globales: capitalisme organisé, communisme, technocratie, collectivisme pluraliste, etc.» ${ }^{46}$, lutte dont l'effet va être de provoquer d'âpres conflits «entre les planifications nationales et internationales, faites soit par les trusts et les cartels, soit par les Etats, soit, enfin, par les producteurs et les consommateurs eux-mêmes » (idem). On reconnaîtra l'identification du caractère de plus en plus contraignant des décisions prises à l'échelle internationale, sur les activités locales, ainsi que l'identification d'une pluralité de types d'acteurs interagissant dans le champ de l'international ou du supranational (l'auteur anticipe sur l'intervention des ONG).

Contrairement à R. Aron, G. Gurvitch note « une tendance vers le 'dépècement de la souveraineté de l'Etat', sous l'action des entreprises organisées en cartels, des syndicats de salariés et d'employeurs, des règles produites par la négociation collective dont il est exclu, sans oublier l'activité intense des partis politiques ${ }^{47}$.

Au travers de ces quelques exemples, il devient clair que la société-monde a déjà rencontré l'intérêt de la sociologie qui a su, sinon forger à son endroit des concepts sur mesure, tout au moins produire des problématiques et esquisser des hypothèses, dont une caractéristique est de refléter l'époque de leur conception (Première Guerre Mondiale pour les fondateurs, Guerre Froide pour les derniers auteurs cités). Certains ont souligné la menace que représentait la perspective d'une humanité réellement unifiée, tandis que d'autres en anticipaient le nécessaire avènement. Risques liés à l'indépendance des multinationales ou à l'impossibilité de construire une identité contre une altérité disparue, rôle central de l'acteur étatique dans l'élaboration du supranational ou caractère automatique du mouvement d'unification planétaire, le spectre des regards sociologiques portés sur le monde a, depuis longtemps, présenté une grande richesse.

\section{ANALYSER L'OBJET-MONDIALISATION: IDENTIFIER LES STRUCTURES DU DÉSORDRE}

Le terme de mondialisation, par son suffixe, indique bien l'idée d'évolution, de modification, plus précisément une modification caractérisant l'ensemble des sociétés dont les spécificités s'estomperaient et l'interdépendance s'accroîtrait. Vue sous cet angle, l'analyse de la mondialisation constitue bien une forme particulière d'analyse du changement social. On peut alors souligner combien la sociologie est légitime dans cette perspective, puisque cette discipline est née de la volonté de comprendre les transformations sociales dont les manifestations s'avéraient, depuis la fin du XVII ${ }^{e}$ siècle en Europe, de plus en plus inquiétantes. Comme le souligne G. Bouthoul, «Aussi loin que l'on remonte parmi les précurseurs de la sociologie, on constate que les progrès marquants, accomplis dans le domaine de la réflexion sur les phénomènes sociaux, ont vu le jour en période de crise ou à propos d'une crise ${ }^{48}$. Cette volonté de comprendre était, de plus, liée à

\footnotetext{
46 G. Gurvitch, 1968, p. 3.

47 G. Gurvitch, 1968, pp. 498-499.

48 G. Bouthoul, 1949, p. 7.
} 
une volonté de maîtriser le cours des événements. En sorte que, chez les premiers sociologues, la conception du changement social est inséparable d'une conception préalable de la société idéale (désirable ou raisonnable).

A partir de ce projet initial, de nombreuses approches du changement ont été développées. Certaines postulent l'existence de tensions micro-sociales: entre partie socialisée et asociale chez G. Simmel (1981), socialisation primaire et secondaire chez P. Berger et T. Luckman (1986), logiques d'actions irréductibles chez F. Dubet (1994). D'autres sociologues privilégient les tensions macrosociales: tension entre solidarité mécanique et progrès de la division du travail (Durkheim, 1978b); lutte pour le contrôle de l'historicité (A. Touraine, 1993), lutte pour la préservation/amélioration de sa position (P. Bourdieu, 1980). L'œuvre d'Alain Touraine illustre particulièrement le primat de la tension: «le conflit n'est ni contradiction ni révolte, mais forme sociale de l'historicité, de la production de la société par elle-même ${ }^{49}$. Pour l'auteur: «la production de la société par elle-même se réalise à travers un conflit central, de sorte que la modernité est toujours divisée entre des adversaires dont aucun n'est guidé seulement par la raison et l'autre seulement par la tradition ou la passion.» ${ }^{50}$

A ces courants s'opposent ceux pour qui le social est en construction permanente sous l'effet de la recherche de compromis, de la négociation et de la coopération: sociologie compréhensive (Weber, 1995), individualisme méthodologique (R. Boudon, 1984), théorie de la régulation (J.-D. Reynaud, 1993). Cette différence se double d'une opposition entre ceux qui privilégient, dans l'analyse du changement, les acteurs porteurs d'une innovation, d'abord culturelle (l'élite), et ceux qui l'envisagent comme résultant d'une logique systémique (automatisme social, selon l'expression de P. Naville, 1963).

Si les théories du changement partent d'une transformation empirique, pour en comprendre la genèse, les théories de la modernisation empruntent le chemin inverse: partant de volontés explicites de modifier le social (réforme par exemple), elles en étudient les conséquences concrètes et, de là, tentent de rendre compte du résultat, soit en pointant la responsabilité des acteurs en situation (D. Linhart, 1994), soit en soulignant le rôle des représentations ou des intentions (D. Martin, 1998). Ces études montrent la nécessité d'analyser la situation de ceux qui n'ont pas présidé à la conception des changements volontaires: peuvent-ils y trouver les ressources d'une action stratégique, sont-ils cantonnés à une position d'exécutant du changement (ou «non-acteur», pour parler comme Dominique Martin), quelles sont leurs capacités de protestation?

Une troisième façon d'aborder, sociologiquement, la question des transformations sociales est de les envisager comme partie prenante de tout phénomène social. Dans cette perspective, s'inscrit G. Bouthoul (1949) qui envisage les sociétés comme en proie au changement permanent, quel que soit son apparent degré d'immobilisme ou de dynamisme. Chaque composante du social («élément de structure») avance à son rythme, rythme lui même variable selon les époques. Il en résulte un décalage entre les secteurs d'une même société, ce qui engendre un système de tensions qui s'équilibrent. L'auteur parle alors d'enchevêtrement, de combinaisons de forces

\footnotetext{
A. Touraine, 1984, pp. 139-140.

50 A. Touraine, 1993, p. 11.
} 
sociales, mais également de dépendances mutuelles entre les différents éléments de structure. Pour l'auteur, la notion d'équilibre doit avant tout être considérée comme une méthode d' analyse des faits sociaux, qui permet de souligner que toute inhibition des forces de ré-équilibrage conduit à la démesure, la crise. C'est ici souligner l'importance des forces de résistances qui empêchent les dérives.

A la même époque, G. Gurvitch définit le social comme constitué d'une multitude de plans, entre lesquels s'instaure une dialectique, « un va-et-vient perpétuel qui tisse la trame même de la réalité sociale $»^{51}$. En effet, la réalité sociale «s'affirme d'abord et avant tout dans les «phénomènes sociaux totaux » ou totalités en marche - ces foyers d'ébullition volcanique compris dans un mouvement de flux et de reflux (...) pour lesquels les Nous, les groupes et les sociétés se créent et se modifient eux-mêmes $»^{52}$. Au-delà de ce vocabulaire imagé, l'auteur propose une conception du changement social comme émergeant des «conduites collectives dérogatoires, novatrices, qui modifient les pratiques et les modèles, et créent des symboles nouveaux ». Mieux, «tout ce qui a été fait antérieurement, tout ce qui est acquis, stabilisé, cristallisé dans la réalité sociale, se dresse devant elles comme un obstacle à vaincre $»^{53}$.

En d'autres termes, il existe une dynamique interne au social, qui lui est consubstantielle, présente dans toute société, où «à tout moment de leur existence, un drame aigu se joue entre les forces de conservation et les forces d'innovation (...), entre la révolution «permanente » et la contre-révolution non moins permanente ${ }^{54}$. Quant à l'identification de structures, elle n'a rien d'incompatible avec l'analyse du changement, du fait que, au sein de chaque société, selon les groupes ou les phénomènes, quatre types de temporalités se rencontrent: temporalité en avance par rapport à la société; temporalité en retard; alternance entre retard et avance; moments de crises $^{55}$. Pour G. Rocher, «toute organisation sociale, celle particulièrement des sociétés complexes, comporte en effet diverses contradictions de structure, sources constantes de frictions et de conflits $»^{56}$. Ces contradictions proviennent du fait que chaque structure est constituée d'éléments d'âge différents, et que chaque groupe social «a sa perspective propre sur la société, par la suite de la place qu'il y occupe». L'irréductibilité des perspectives créerait une situation potentiellement conflictuelle, source de changement et chaque changement, loin de résoudre les conflits, en engendrerait de nouveaux.

Prolongeant cette perspective, G. Balandier (1988) suggère d'analyser le social avant tout comme «du désordre», «du mouvement et des fluctuations ». Plus précisément, l'étude du social doit prendre en compte l'existence de temporalités différentes: certains «secteurs » n'évoluent que lentement, d'autres beaucoup plus rapidement, d'autres encore se maintiennent dans la répétition; ces évolutions n'ont, de plus, pas la même «direction». Il en résulte que, sous l'ordre apparent des sociétés, travaillent des discordances et des désajustements. Ces derniers sont sans doute d'autant plus sensibles que la «mise en relation des

\footnotetext{
G. Gurvitch, 1968, p. 8.

G. Gurvitch, 1967, p. 20.

G. Gurvitch, 1968, p. 101.

G. Gurvitch, 1968, p. 103.

55 G. Gurvitch, 1967, p. 213.

56 G. Rocher, 1968, p. 123.
} 
sociétés s'est généralisée depuis peu ». Le mode normal d'existence du social est fait de l'interaction permanente entre ordre et désordre. L'auteur met toutefois en garde contre cette approche, qui peut conduire à «abandonner la société à ellemême et à tout miser sur le retour de l'individu ou de l'acteur, sur la vertu de l'initiative ou de la spontanéité».

Avec la notion de dyschronie, N. Alter (2001) retrouve ce courant d'analyse, en soulignant combien les phénomènes sociaux résultent d'un enchevêtrement de processus complexes, jamais achevés, jamais assurés et non linéaires, susceptibles de régressions, n'évoluant pas à la même vitesse, où des acteurs sont aux prises avec les formes cristallisées d'actions passées.

Il existe donc une riche tradition d'analyse du changement social dont peut profiter l'étude de l'objet mondialisation. Ainsi, l'observateur peut envisager la période contemporaine sur le modèle de la tension entre temporalités distinctes. Cela lui permettra, d'une part, de prendre en compte les complexes articulations entre les modifications des identités (culturelles, professionnelles, religieuses) et les transformations survenues dans le domaine des techniques ou dans le champ économique ${ }^{57}$. Mais cela permettra également de rapporter les apparentes mutations récentes (la dérégulation-rerégulation des économies des années 1980, par exemple) à des projets et à des pratiques plus anciens dont l'existence était provisoirement passée au second plan, mais dont la répétition têtue, l'accumulation, a fini par porter ses fruits (permanence de l'idéologie libérale, résistance à des projets d'émancipation sur fond de lutte contre le communisme). Par ailleurs, raisonner en termes de temporalités distinctes (à l'échelle mondiale) n'a de sens qu'à condition d'y voir le travail jamais achevé de collectifs aux ressources profondément inégales. Ce qui conduit à privilégier l'examen du réel comme conséquence de décisions particulièrement structurantes, prises par des acteurs identifiables, conséquences qui renforcent parfois leurs capacités d'action. Cette posture présente alors l'avantage de distinguer plusieurs niveaux de responsabilités dans la production de la société (ou son auto-institution) tout en laissant la possibilité d'imaginer d'autres possibles.

Il reste à aborder la question de l'interaction entre sciences sociales et société-monde, à la fois pour mesurer l'ampleur du travail de distanciation que doit opérer l'observateur «savant» et pour apprécier la contribution de son discours au mouvement même de la mondialisation.

\section{SOCIÉTÉ-MONDE ET TRANSFORMATION DES SCIENCES SOCIALES}

En effet, on peut se demander quelle conséquence peut avoir l'interdépendance croissante des sociétés à l'échelle mondiale, sur notre capacité à rendre compte précisément du phénomène. Rappelons qu'en conclusion des Formes élémentaires de la vie religieuse, où $\mathrm{E}$. Durkheim réfléchit à l'origine sociale de la notion de catégories de la pensée, Durkheim examine «le concept même de totalité». Ce concept constitue «la catégorie par excellence» et «la forme abstraite du concept

57 C'est, notamment, l'une des postures adoptées par M. Castells (1998 et 1999). 
de société ${ }^{58}$. Et si cette catégorie est d'abord marquée du sceau de chaque société particulière où elle émerge, «elle tend de plus en plus à se débarrasser des éléments subjectifs et personnels qu'elle charrie encore à l'origine». Grâce à «cette vie internationale qui a déjà pour effet d'universaliser les croyances religieuses (...) la société cesse d'apparaître comme le tout par excellence, pour devenir la partie d'un tout beaucoup plus vaste, aux frontières indéterminées et susceptibles de reculer indéfiniment $\gg^{59}$. En d'autres termes, c'est parce que les individus ont progressivement pris conscience du contexte macro-social dans lequel leur société était plongée (les relations internationales) que s'est progressivement dégagé le concept universel de totalité. En prenant conscience de l'universel la pensée s'universalise (les différentes formes locales d'un même concept s'unifient).

Plus récemment, N. Elias insiste sur le fait que notre capacité à analyser les relations sociales au plan inter-étatique, cet «espace social sans contrôle efficace de la violence $»^{60}$, est limitée par le développement de ces relations. En effet, nous sommes soumis à une double contrainte: d'un côté, au sein d'un Etat national, «il est strictement interdit d'attaquer d'autres hommes avec violence et de les tuer»; de l'autre, au niveau des relations interétatiques, «cela passe pour un devoir de développer, entretenir et utiliser une capacité de violence à l'égard d'autres hommes ${ }^{61}$. Sous cette double contrainte, les individus «vivent avec deux codes de comportement distincts et opposés». Aussi, faute d'une distanciation suffisante, savants ou profanes considèrent le plus souvent les rapports sociaux de niveau inter-étatique, comme résultant d'intentions individuelles (ce sont les dirigeants qui veulent la paix ou la guerre), alors que, selon N. Elias, ces rapports résultent également des structures et de la dynamique propre au champ international (hiérarchies, positions, capacité à exercer la violence, compositions d'alliances et d'allégeance entre Etats, etc.).

De nos jours, on peut constater un parallèle entre, d'une part, la mise en œuvre, à une échelle jusqu'à présent inconnue, du modèle libéral dans le champ de l'économie, et d'autre part, une «fluidification» de la sphère savante: de nombreuses «nouvelles sociologies » ${ }^{62}$ émergent et se diffusent (un peu à la manière des «nouvelles technologies» ou de la «nouvelle économie»); de «nouveaux» concepts sont introduits, mobilisés, répétés, sans que la preuve de leur pertinence ne précède toujours leur emploi. D'une part, une porosité semble être apparue entre les sciences sociales et les sciences de la nature, les premières empruntant aux secondes des paradigmes récents, comme ceux visant à rendre compte du chaos: «la tentation est grande de traduire en langage sociologique le nouveau discours de la nature, en découvrant des isomorphismes entre domaines fort éloignés, en procédant par analogie, en se démarquant des démarches interprétatives.» ${ }^{63}$ Et d'autre part, la doctrine «laisser faire laisser passer» des premiers théoriciens du libre échange semble avoir envahi l'imaginaire et les pratiques d'une profession pourtant vouée à la prise de distance, au recul critique et au scepticisme.

\footnotetext{
58 E. Durkheim, 1979, pp. 629-630.

59 E. Durkheim, 1979, pp. 634-635.

60 N. Elias, 1993, p. 127.

$61 \quad$ N. Elias, 1993, p. 124.

62 Selon l'expression de P. Corcuff (1995).
} 
C'est ce que soutient, notamment, D. Méda (1995) pour qui les sciences économiques se sont, en particulier en France depuis la fin des années 1970, enrichies de nombreux éléments, visant «à corriger les simplifications les plus criantes de la théorie économique standard grâce aux résultats des recherches engagées en sociologie, en droit ou en anthropologie». L'auteur fait référence à plusieurs "'écoles' (dites de la régulation, de l'économie des conventions, de l'économie des institutions)». Mais ces incorporations se sont effectuées «sans changer le moins du monde ses présupposés majeurs », «les travaux des anthropologues sur le don (...) sont ainsi retraités à partir d'hypothèses de la microéconomie, en complète contradiction avec les ambitions et le type d'approche de l'ethnologie et de l'anthropologie». Il y a donc détournement des acquis de la sociologie. Mais réciproquement, cette dernière «destinée, à l'origine, à analyser la société comme un tout, a largement intégré la méthode et les hypothèses de base de l'économie, sans toujours s'en rendre compte, par le simple fait qu'elle recourait massivement à la théorie des jeux ou aux méthodes dites du choix rationnel ${ }^{64}$.

La même critique est formulée par A. Mattelart qui souligne qu'après plusieurs décennies de déréglementations dans les domaines économiques et financiers, «les usages acritiques de termes promus ou revisités à la faveur du libre-échange ont proliféré », on « assiste à une véritable déréglementation des univers conceptuels qui nous servent à désigner le monde ${ }^{65}$. Citons cette « façon de mettre sur le même plan les acteurs sociaux et les objets (...) les humains et les non-humains », approche qui contribue, sous la façade d'un discours logique, axiomatique, «à faire disparaître tout ce qui a fait les grandes heures de la sociologie classique et même les interrogations de notre époque. (...) A la place, on a affaire à des agents sans épaisseur spécifique (...) dotés seulement de manières de réagir à des situations, passant d'un état à l'autre, d'une façon aussi fluide que dans le cas des marchés parfaits ${ }^{66}$. Q. Delaunay fait ici référence à la «nouvelle sociologie des sciences» de Bruno Latour et Michel Callon (appelée également constructivisme radical), ainsi qu'à l'économie des conventions, deux courants typiques des années 1980-1990, en ce qu'ils incarnent la volonté de «remise en cause de la sociologie constituée (Weber, Marx, Bourdieu, Boudon, Elias, Aron, Crozier, Goffman, etc.) et en particulier la remise en cause des structures sociales dans l'explication de l'activité des individus ${ }^{67}$. D'autres critiques peuvent être mobilisées dans le même sens: ainsi P. Bourdieu (2001) et P. Dubois (1999) fournissent une critique argumentée des fondements de la nouvelle sociologie des sciences ${ }^{68}$, tandis que J.-R. Tréanton (1993) souligne les nombreuses faiblesses conceptuelles de l'économie des conventions.

G. Balandier, 1988, p. 84.

D. Méda, 1995, pp. 256-258.

A. Mattelart, 1999, p. 351.

Q. Delaunay (1997).

Q. Delaunay, pp. 193-194.

68 La seule observation de laboratoires, même pendant deux ans, «constitue une source d'aveuglement quant aux processus à l'œuvre hors du laboratoire qui permettent d'expliquer la formation d'un consensus autour de la validité de telle ou telle connaissance» (M. Dubois, 1999, p. 49). Tout compte fait, «derrière l'abstraction d'une rhétorique de 1' actant', des 'humains' et des 'non-humains', Callon et Latour proposent (...) la théorie de l'acteur-réseau [qui] n'est rien d'autre qu'une régression vers une description réaliste» des situations d'invention (M. Dubois, 1999, p. 64). 
Ces tendances constituent simultanément une euphémisation: «ces concepts inspirés par les pratiques marchandes jouent le rôle d'écran par rapport à la mise en place de nouvelles 'sociétés de contrôle'» ${ }^{69}$. En fait, celles-ci ne sont pas évidentes à discerner, mais elles s'inspirent du modèle d'organisation de l'entreprise en réseau (la firme globale, flexible), qui laisse croire que les raisons des conflits (notamment hiérarchiques) ont été éliminées. Et dans ce travail d'euphémisation des conditions de domination, les sciences sociales, certes instrumentalisées, mais avec le concours de scientifiques, jouent un rôle, «dans la mesure où, pour pouvoir fonctionner, ces nouveaux schémas d'efficacité et de légitimité ont besoin d'une représentation constructiviste de la société. Celle-ci a notamment été élaborée dans le savoir expert des sociologues, depuis les années 1960, et largement diffusée dans les organisations, au point de modifier les catégories de perception des acteurs sociaux eux-mêmes $»^{70}$. Car selon cette conception, «il ne reste plus alors que des connexions, des négociations et des interactions toujours réalisées dans des situations particulières ${ }^{71}$. Au lieu de 《voir le monde comme un espace-temps où coexistent des formes d'exploitation, d'oppression et d'humiliation à la fois anciennes et nouvelles, ainsi que des formes de résistance à la fois éprouvées et inédites $\gg^{72}$, ces courants ne retiennent qu'un mode d'explication du social: les relations marchandes.

Analysant les transformations récentes de la sociologie religieuse et de la sociologie urbaine, L. Voyé (2001) montre comment ces évolutions présentent des analogies avec celles touchant la société dans son ensemble. Ainsi, le champ du religieux est envisagé comme «ouvert au choix d'un marché libre». L'étude des villes incorpore le concept d'espace virtuel et analyse leur fonctionnement en termes de rencontre entre espace des flux et espace des lieux. Ce qui montre «combien la tentation est grande de penser prioritairement la sociétémonde à travers des références de nature économique ${ }^{73}$.

L. Voyé va plus loin en notant que la mondialisation influence également le fonctionnement de la discipline: dorénavant, on en arrive à «apprécier la recherche en termes de 'production' plutôt que comme une œuvre de savoir», ce qui conduit les chercheurs à accorder une importance démesurée à la quantité d'ouvrages, d'articles et, conséquemment, à être «tentés de reproduire, sous des modes divers, une pensée qu'ils ont émise une fois pour toute», évitant de la soumettre à un réel débat.

Finalement, l'intérêt pour l'objet mondialisation souligne l'urgence de discuter/critiquer la pertinence (ou le domaine d'usage) de certaines théories surgies en même temps que le phénomène lui-même (la «mondialisation») prenait une ampleur inédite. Il montre notamment que, derrière leur apparente neutralité, ces courants contribuent à une remise en cause de certains fondements de la sociologie: ils font fi des inégalités multidimensionnelles entre acteurs, de leur inégal accès au contrôle des structures (et donc, de leur inégale responsabilité

\footnotetext{
69 A. Mattelart, 1999, p. 352.

70 J. de Munck, 2001, p. 127.

71 J. de Munck, 2001, p. 130.

72 A. Mattelart, 1999, p. 376.

73 L. Voyé, 2001, p. 290.
} 
dans la production du social); ils négligent le poids des décisions étatiques, naturalisant l'arrière-fond culturel et idéologique qui produit et encadre, aussi bien le «marché» que les procédures d'arbitrage. Simultanément, ils contribuent à donner corps aux phénomènes qu'ils entendent négliger (rapports de domination, interdépendances macrosociales).

\section{SOCIOLOGIE DE LA MONDIALISATION ET OCCIDENTALISATION DES SCIENCES SOCIALES}

Mais la mondialisation engendre d'autres effets sur notre capacité à l'analyser. Nous pensons ici aux rapports de dominations entre nations, appliqués à la sphère des sciences sociales. R. Aron soulignait déjà que «les concepts de la sociologie américaine, en passe de devenir ceux de la sociologie mondiale (pays d'obédience marxiste-léniniste exclus), s'appliquent à l'homme social en tant que tel, quelle que soit son activité, quelle que soit l'organisation à laquelle il appartient $»^{74}$.

Trente ans plus tard, A. Sen peut souligner que «la focalisation sur des ouvrages appartenant à une seule culture entrave toute liberté de découvrir d'autres idées ${ }^{75}$. Il faut en effet se demander dans quelle mesure les productions intellectuelles non américaines et non occidentales ont quelque chance d'influer sur notre compréhension du monde, dans un espace savant de plus en plus susceptible de s'internationaliser. Pour le moment, note A. Appadurai (1999), l'internationalisation des sciences sociales consiste, à «améliorer la façon dont les autres appliquent nos préceptes», effort pédagogique dont le principal effet est de «creuser l'écart» entre cette conception de la recherche et «les traditions plus générales de critique et de discussion en matière sociale».

Sans approfondir ici cette question, on peut remarquer avec l'auteur que, par essence générateur d'inégalités, le mouvement de mondialisation des économies «entraîne une fragmentation et une répartition inégale des ressources nécessaires » à son étude et donc, entrave sérieusement les coopérations susceptibles d'en «faciliter la compréhension ou la critique». Il n'est pas jusqu'à l'imagination du «savant» qui ne soit sous l'emprise des codes dominants et donc, subisse les conséquences de la mondialisation (notamment, via les flux médiatiques mondialisés). De plus, comme le montre O. Kozlarek (2001), la principale faiblesse des sciences sociales, même quand elles se prétendent critiques, est de s'inscrire dans le projet européen de construire un cosmos, une cosmopolis caractérisée par la centralité de l'Europe, dont la mondialisation contemporaine peut être considérée comme une conséquence.

Une manière de mettre à distance ces formes de socio-centrisme pourrait consister à confronter les différentes conceptions sociologiques de l'objet-monde, en prenant soin de n'écarter aucune ère géographique, ce qui, dans une large mesure, reviendrait à examiner - pour les comparer et en apprécier la pertinence, la complémentarité et le domaine d'usage - les différentes conceptions de l'universel. Ce qui requiert des intéressés qu'ils cherchent à se rapprocher du modèle

\footnotetext{
74 R. Aron, 1969 , p. 6.

75 A. Sen,
} 
idéal de communauté de communication sans distorsion élaboré par J. Habermas (1987), de manière à ne pas reproduire, dans l'espace même de la discussion, les rapports de domination qu'il est question de dépasser.

\author{
Laboratoire de Sociologie du Changement des Institutions \\ et Groupe de Recherches interdisciplinaire \\ sur les organisations et le travail \\ France Télécom $R \& B$ \\ Issy Moulineaux, France
}

\title{
BIBLIOGRAPHIE
}

N. Alter, L'innovation ordinaire, PUF, 2001.

A. Appadurai, Mondialisation, recherche, imagination, in «Revue internationale des sciences sociales », juin, n 160, 1999, pp. 257-267.

R. Aron, La lutte des classes. Nouvelles leçons sur les sociétés industrielles, Gallimard, 1964 [1956-1957].

R. Aron, Paix et guerre entre les nations, Calman-Lévy, 1984 [1962].

R. Aron, Les désillusions du progrès, Calman-Lévy, 1969 [1965].

R. Aron, Les étapes de la pensée sociologique, Gallimard, 1967.

G. Balandier, Le désordre. Eloge du mouvement, Fayard, 1988.

P. Berger et T. Luckman, La construction sociale de la réalité, Méridiens Klincksieck, 1986 [1966].

R. Boudon, La place du désordre, Critique des théories du changement social, PUF, 1984.

P. Bourdieu, Le sens pratique, Paris, Minuit, 1980.

P. Bourdieu, Science de la science et réflexivité, Raisons d'agir, 2001.

G. Bouthoul, Traité de sociologie, 2 tomes, Payot, 1949 [1946].

G. Busino, Raymond Aron et la sociologie, in «L'Année sociologique», n 36, 1986, pp. 291-315.

G. Busino, Quelles significations attribuer aux processus de rationalisation de la mondialisation?, in Daniel Mercure (dir.), Une société monde? Les dynamiques sociales de la mondialisation, De Boeck Université et Presses Universitaires de Laval, 2001, pp. 167-181.

M. Castells, La société en réseaux, 3 tomes, Fayard, 1998 et 1999.

F. Cooper, Le concept de mondialisation sert-il à quelque chose? Un point de vue d'historien, «Critique internationale», $\mathrm{n}^{\circ} 10$, janvier, 2001, pp. 101-124.

P. Corcuff, Les nouvelles sociologies, Nathan, 1995.

Q. Delaunay, Le conventionnalisme, in J.-P. Durand et R. Weil, Sociologie contemporaine, Vigot, 1997, pp. 185-198.

F. Dubet, Sociologie de I' expérience, Le Seuil, 1994.

M. Dubois, Introduction à la sociologie des sciences. PUF, 1999.

E. Durkheim, Le socialisme. Sa définition. Ses débuts. La doctrine saint-simonienne, Retz-CEPL, 1978a [1895-6].

E. Durkheim, Textes, $t$. 1 : éléments d'une théorie, Minuit, 1975 [1887-1917].

E. Durkheim, Textes, t. 3 : fonctions sociales et institutions, Minuit, 1975 [1887-1917].

E. Durkheim, De la division du travail social, PUF, 1978b [1930].

E. Durkheim, Les formes élémentaires de la vie religieuse. Le système totémique en Australie, PUF, 1979 [1912].

N. Elias, La civilisation des mœurs, Calman-Levy, 1973 [1939].

N. Elias, La Dynamique de l'occident, Presses Pocket, 1975 [1939].

N. Elias, Engagement et distanciation, Fayard, 1993 [1983].

Y. Goussault et A. Guichaoua, Sciences sociales et développement, A. Colin, 1993. 
G. Gurvitch, La vocation actuelle de la sociologie, 2 tomes, PUF, 1968 [1950].

G. Gurvitch, Traité de sociologie, 2 tomes, PUF, 1967 [1958].

J. Habermas, L'agir communicationnel, t. I, Fayard, 1987 [1981].

O. Kozlarek, Critical theory and the challenge of globalization, in «International Sociology», vol. 16(4), décembre 2001, pp. 607-622.

$\mathrm{S}$. Latouche, La mondialisation démystifiée, in $\mathrm{E}$. Goldsmith, J. Mander (dir.), Le procès de la mondialisation, Fayard, 2001.

D. Linhart, La modernisation des entreprises, La Découverte, 1994.

D. Martin, La modernisation dans la crise: de Talcott Parsons à Jurgen Habermas, in D. Gerritsen et D. Martin (dir.), Effets et méfaits de la modernisation des entreprises dans la crise, Desclée de Brouwer, 1998, pp. 17-46.

D. Martin, J.-L. Metzger et P. Pierre, Les métamorphoses du monde. Pour une sociologie de la mondialisation, Seuil, 2003.

A. Mattelart, La mondialisation de la communication, PUF, 1996.

A. Mattelart, Histoire de l'utopie planétaire, La Découverte, 1999.

M. Mauss, Euvres, t. 3, Minuit, 1969.

D. Méda, Le travail, une valeur en voie de disparition, Aubier, 1995.

D. Mercure, Une société monde?, in Daniel Mercure (dir.), Une société monde? Les dynamiques sociales de la mondialisation, De Boeck Université et Presses Universitaires de Laval, 2001, pp. 9-16.

J. de Munck, Pour une critique de la raison procédurale, in Daniel Mercure (dir.), Une société monde? Les dynamiques sociales de la mondialisation, De Borck Université et Presses Universitaires de Laval, 2001, pp. 115-132.

P. Naville, Vers l'automatisme social?, Gallimard, 1963.

T. Parsons, Sociétés. Essai sur l'évolution comparée, Dunod, 1973a [1966].

T. Parsons, Le système des sociétés modernes, Dunod, 1973b [1971].

P. Raynaud, Max Weber et les dilemmes de la raison moderne, PUF, 1987.

J.-D. Reynaud, Les règles du jeu, Armand Colin, 1993 [1989].

G. Rocher, Introduction à la sociologie générale, t. 3, Le changement social, Seuil, 1968.

G. Rocher, La mondialisation est un phénomène pluriel, in Daniel Mercure (dir.), Une société monde? Les dynamiques sociales de la mondialisation, De Boeck Université et Presses Universitaires de Laval, 2001, pp. 17-31.

J.-C. Ruano-Borbalan, Les orientaux sont-ils faits pour le capitalisme?, in «Sciences humaines", $\mathrm{n}^{\circ} 118$, juillet, 2001, pp. 45-49.

A. Sen, La Raison, l' Orient et l'Occident, in «Esprit», 12, 2000, pp. 80-98.

G. Simmel, Comment les formes sociales se maintiennent, in Sociologie et épistémologie, PUF, 1981 [1896-97], pp. 171 -206.

G. Simmel, Philosophie de l'argent, PUF, 1987 [1900].

W. Sombart, L'apogée du capitalisme, tome 2, Payot, 1932 [1921].

W. Sombart, Le bourgeois, Payot, 1966 [1928].

A. de Swaan, Pour une sociologie de la société transnationale, in « Revue de synthèse », $\mathrm{n}^{\circ}$ 1, 01-03, 1998, pp. 89-111.

A. Touraine, Production de la société, Seuil, 1993 [1973].

A. Touraine, Le retour de l'acteur, Fayard, 1984.

J.-R. Tréanton, Tribulations de la justice, in « Revue française de sociologie », n 4, 1993, pp. 627-655.

L. Voyé, La société monde: un défi pluriel pour la sociologie, in Daniel Mercure (dir.), Une société monde? Les dynamiques sociales de la mondialisation, De Boeck Université et Presses Universitaires de Laval, 2001, pp. 285-302.

M. Weber, Histoire économique. Esquisse d'une histoire universelle de l'économie et de la société, Gallimard, 1991 [1923].

M. Weber, Economie et Société, Plon, 1995 [1956]. 